\title{
Agent-Based Modeling of Harvest Decisions by Small Scale Forest Landowners in Maine, USA
}

\author{
Jessica E. Leahy, ${ }^{1}$ Erika Gorczyca Reeves, ${ }^{1}$ Kathleen P. Bell, ${ }^{2}$ \\ Crista L. Straub, ${ }^{3}$ and Jeremy S. Wilson ${ }^{4}$ \\ ${ }^{1}$ School of Forest Resources, University of Maine, 241 Nutting Hall, Orono, ME 04469, USA \\ ${ }^{2}$ School of Economics, University of Maine, 200 Winslow Hall, Orono, ME 04469, USA \\ ${ }^{3}$ Sustainability Solutions Initiative, University of Maine, 104 Norman Smith Hall, Orono, ME 04469, USA \\ ${ }^{4}$ Harris Center for Conservation Education, 83 King's Highway, Hancock, NH 03449, USA
}

Correspondence should be addressed to Jessica E. Leahy; jessica.leahy@maine.edu

Received 15 February 2013; Accepted 3 June 2013

Academic Editor: Audrey L. Mayer

Copyright (C) 2013 Jessica E. Leahy et al. This is an open access article distributed under the Creative Commons Attribution License, which permits unrestricted use, distribution, and reproduction in any medium, provided the original work is properly cited.

\begin{abstract}
Small-scale forests are an excellent example of coupled social-ecological systems, which involve human and biophysical subsystems with complex two-way feedback interactions. The multifaceted nature of landowner decisions drives a significant need to better understand decision-making processes, reactions to policy, and combined impacts on ecosystems in a comprehensive manner. Small-scale forests require an integrated approach to modeling the social and biophysical components comprehensively. Agentbased modeling involves modeling individualistic behavior and interpreting patterns that emerge. The interaction between agents and their environments makes this a valuable tool to assess repeated decisions of individual landowners responding to changing environmental conditions. Agent-based models can be used to determine potential ecological, economic, and social outcomes of landowner decisions and reactions to changing conditions. A forest landowner agent-based model experiment was developed to model timber harvesting in Maine, USA. We present baseline simulation results and compare the effect of a social change (an increased tax rate) and a biophysical change (a pest outbreak resulting in increased tree mortality) on the system. These three scenarios were analyzed using ANOVA and MANOVA tests on harvested hectares and landowner goal scores to assess landowner behavior and priorities by action. We conclude by reviewing implications for future modeling efforts.
\end{abstract}

\section{Introduction}

Managed small-scale forests are an excellent example of a coupled social-ecological system (SES). An SES is the one that joins human and biophysical subsystems in two-way feedback interactions [1]. Small-scale forests are best described as an SES because of the dynamic interactive feedback loops between the social aspect of landowners and the biophysical components of the forest itself. These systems are dynamic, intricate, and complex to study, model, and manage. Smallscale forest landowners have become a dominant form of landownership in the United States, accounting for 106 million hectares. Much of these productive forests are held in northeast USA where nearly 38 million hectares of forested land are held by 4.8 million small-scale forest landowners $[2,3]$. Small-scale forests are generally considered to be privately-owned forest parcels ranging between 4 and 405 hectares in size. Due to their nature, researchers need a new strategy to study these coupled systems-an integrated modeling approach - to best represent the multifaceted aspects of landscapes dominated by forest ownerships.

Maine alone is estimated to have 2.3 million hectares of forested land belonging to 88,000 small-scale forest landowners [4]. Productive forests have a large impact on the state's economy [5]. With over $90 \%$ of 8 million hectares of Maine in forest cover, it is the most heavily forested state in the United States [6]. Furthermore, 97\% of Maine's forests are classified as productive timberland, $95 \%$ of which is privately owned $[7,8]$. Small-scale forests account for approximately $34 \%$ of the forest area in Maine and about 25\% of timber harvested each year [5]. Maine possesses nearly half of all woody biomass in the northeast. Positive net growth of timber on privately owned properties is second only 
to nongovernmental organizations and tribal landowners. Particularly with interest in the biomass and bioproducts markets, small-scale forest landowners will be looked upon to supply timber and fiber.

We chose to focus on small-scale forest landowner timber harvesting behavior. Understanding landowner decisions about whether to harvest their trees and supply timber to the forest products market is essential because of its economic and ecological consequences. While timber harvesting represents a significant economic value in Maine, landowner harvest behavior does not always follow economic or price considerations $[9,10]$. Prediction of smallscale forest timber harvesting trends has remained elusive. Multiple researchers have had difficulty associating smallscale forest motivations with behavior [11-14]. Erickson et al. [15] found that landowner behavior was largely unrelated to economic circumstances, such as timber prices. The disparity of landowner harvesting intentions and actions has resulted in behavior that is difficult to predict. Furthermore, these actions suggest that there may be other motivations for harvesting rather than solely a landowner's management beliefs. Landowner attitude surveys documented low priority of timber harvesting and higher rated objectives of wildlife, recreation, and scenery $[16,17]$. Several studies have found a disconnect between landowner intention to harvest and actual behavior [11-14]. For instance, Massachusetts smallscale forest landowners were found to have high responses of wildlife, recreation, and scenery objectives instead of harvesting [18]. Despite this, researchers found that $84 \%$ of harvested hectares occurred on small-scale forests in a region of Massachusetts [19]. If small-scale forest landowners were truly disinterested in harvesting we would expect a much smaller percentage of harvests occurring on small-scale forests. This suggests harvesting decisions rely on more than management beliefs and objectives. As a result of this seemingly erratic behavior, researchers need a new technique to better represent and understand landowner motivations and actions.

Throughout past and present attempts to understand forest management decisions, many different modeling methods have been employed. On the biophysical side, analysis techniques often include demand and supply projections, forest inventory analysis (FIA), and growth models at the regional or state level. These are used to estimate available timber resources, determine forest trends, and even forecast timber harvesting. In contrast, the social science dimension of smallscale forest research has been limited to surveys, focus groups, and interviews. These often utilize economic analysis, social theory and policy analysis, and occur at regional or state levels $[20,21]$. One key data source is the National Woodland Owner Survey (NWOS) administered through the USDA Forest Service. This survey is used to better understand private forest landowners as part of the Forest Inventory and Analysis (FIA) program, including information such as demographics, attitudes, forest use and management, concerns, and intentions. This annual survey initiated in 2002 compliments the FIA biological forest sampling [2]. Although the NWOS attempts to present both characteristics, the national survey is not necessarily a representative sample that can be used to extrapolate management decisions and policies applicable to smaller scale issues such as statewide, county, or local town management.

Agent-based modeling (ABM) offers an integrated approach to modeling social and ecological systems. In our ABM, social and biophysical modeling approaches were combined. Our objectives are to, first, present an ABM of Maine small-scale forest landowners according to the ODD protocol set by Grimm et al. [22, 23]. Second, we employ two system-wide shocks on the ABM: a social change (increased taxes) and a biophysical change (a disturbance event with high tree mortality). These two scenarios are compared to the baseline to better understand small-scale forest timber harvesting behavior and assess the potential of ABMs for use in small-scale forest policy decisions. The complex nature of forest landowner beliefs and perceptions, along with limitations and opportunities of their land base, drives a significant need to better understand decision making processes, reactions to policy, contributions of resource conditions, and combined impacts on ecosystems and other inadvertent consequences in a comprehensive manner.

\section{Literature Review}

Although small-scale forests are one example of countless worldwide social-ecological systems, they are especially suited for generative social science approaches such as agentbased modeling. Generative social science provides a new strategy to identify societal patterns and norms through the use of simulating individual behavior from the base-level up [24]. As a method of generative social science, agent-based modeling $(\mathrm{ABM})$ is a tool that could further understand coupled social-ecological systems. Agent-based models are “... computationally intensive dynamic simulation model(s) of how individual agents (typically using simple behavioral rules) interact with their environment and each other, giving rise to system-wide macro patterns or emergent properties which cannot be deduced from the individual agent's rules" [25]. The interaction between agents and their environments makes $\mathrm{ABM}$ a valuable tool to assess repeated decisions of individual landowners. ABM can be utilized to determine potential ecological, economical and social outcomes of landowner decisions and reactions to changing policies and conditions. Furthermore, ABM can be used as a learning tool for researchers and policy makers.

Societal patterns can be very difficult to model within the relatively small range of social science techniques commonly implemented (e.g., surveys, focus groups, and interviews). The majority of past small-scale forest research focuses solely on either inductive- or deductive-based science; however, generative social science is progressively being tested by researchers. Essentially the concept is to determine how societal patterns and norms arise based on a generative approach. The method is to "Situate an initial population of autonomous heterogeneous agents in a relevant spatial environment; allow them to interact according to simple local rules, and thereby generate-or "grow"-the macroscopic regularity from the bottom up" [24, page 42]. Epstein [24] presents agent-based modeling as a computational model 
well suited to study this question due to five components: heterogeneity, autonomy, explicit space, local interactions, and bounded rationality. Agent-based modeling allows for heterogeneous agents to essentially represent a diverse combination of individuals. Autonomy refers to the lack of "top-down" control over individual behavior, although forest practice regulations can be incorporated into the modeling. Essentially, individuals make individual decisions and act on their own parcels, allowing patterns to coevolve in a "bottomup" fashion. Furthermore, agents act in defined space with specific boundaries, interact with those around or near them, and are bounded by personal rules and information (e.g., no global system-wide expectations, agents act on their own parcels based on individual-level behavioral rules).

Small-scale forests offer an especially challenging investigation, with landowners often possessing very individualistic and divergent management motivations. Based on Epstein's five factors, timber harvesting behavior is a perfect opportunity for agent-based modeling. This conundrum of individualized behavior is best described by Epstein's [24, page 41] generativist's question: "How could the decentralized local interactions of heterogeneous autonomous agents generate the given regularity?" First, small-scale forests offer a myriad of heterogeneity based on individual landowners; they have multiple social and economic factors emanating from diverse backgrounds. Small-scale forest landowners are also autonomous agents in explicit space. Landowners take actions only within their own parcels. In addition, smallscale forest landowners involve dynamic interactions with those around them or within their social networks, such as neighbors, friends, and small-scale forest landowners. Based on Epstein's description, small-scale forests are a good fit for agent-based modeling, with a spectrum of landowner types and individuals that interact and are constrained to actions within their parcel boundaries.

There have been several studies that set a precedent of $\mathrm{ABM}$ in natural resource management. Pahl-Wostl and Hare [26] found ABM to show great potential to improve social learning and a more integrated method of assessment of natural resource management. Land-use changes and their impacts have also been simulated through the adaptive approach of participatory and agent-based modeling [27]. Specifically, Castella et al. [27] created an ABM to study the effect of development on local farm stakeholders in Vietnam. On a larger regional scale, Valbuena et al. [28] employed ABM to explore land use and cover change. Valbuena et al.s [28] case study demonstrated that ABM provides a useful framework for diverse decision-making processes found in social-ecological systems. More specifically, ABM is capable of identifying key parameters and processes underlying adaptive forest management [29]. Gebetsroither et al.s [29] study modeled the use of forest resources through integrated socioeconomic and forest succession submodels.

Although there have been several land use and forest management related agent-based models, few have specifically addressed small-scale timber harvesting and forest management. Landowners, forest resource professionals, and forest policy makers could greatly benefit from ABM due to the ability to combine multiple models and allow interaction between each. As a coupled SES, the complex nature of smallscale forest management suggests that models are missing key interactions that drive decision-making processes. Common forest growth models do not account for the landowner behavior. Likewise, social theories usually do not take into account the biophysical components of small-scale forest land. The ability to model parcel-based biophysical elements as well as socioeconomic factors would be a great advantage for management and policy decisions.

\section{Methods}

To better understand small-scale forest landowner behavior, we created the forest landowner agent-based model experiment (FLAME). This model is presented according to a modified version of overview, design concepts, and details (ODD) protocol $[22,23]$. Due to the complexity of ABM, use of computer implementation and a desire to compare features and functions across agent-based models, it is critical to communicate model descriptions as consistently, effectively, and clearly as possible. The ODD protocol has emerged as the most preferred way of presenting ABMs. It uses a sequentially organized technique of presenting ABMs through seven elements: purpose, state variables and scales, process overview and scheduling, design concepts, initialization, input, and submodels. The code and full documentation are available from the lead author upon request.

3.1. Purpose. The purpose of FLAME was to model complex small-scale forest landowners within a coupled socialecological system. Specifically, this model simulated social and ecological changes over extended periods of time based on landowner decisions. FLAME was designed to simulate the behaviors of small forest landowners within small rural to semirural communities within the state of Maine by focusing on one community. The model consisted of landowners, who in turn possessed land in a mapped landscape that had its own attributes. As in real life, the land attributes, or resources, that were available to a given landowner influenced what they could do with their parcel. These landowners, or agents, within their assigned parcel had the ability to interact with each other as well interact with their parcel. The ultimate goal of this system was to allow agents to autonomously make decisions in an attempt to improve their own welfare. For this study, we focused solely on landowner timber harvesting behavior. Agent decisions are constrained by their beliefs in the techniques available to them as well as their opinion of other agents with whom they have communicated.

3.2. State Variables and Scales. FLAME was broken into two main components, the static and dynamic models. The static model consisted of the landscape and landowner parameters at a given point in time. Information contained in the static model was evaluated at the beginning of each yearly period to drive the dynamic model. The dynamic model was the construct that incorporates all interaction among landowners, actions, and advancement of parameters each year. 


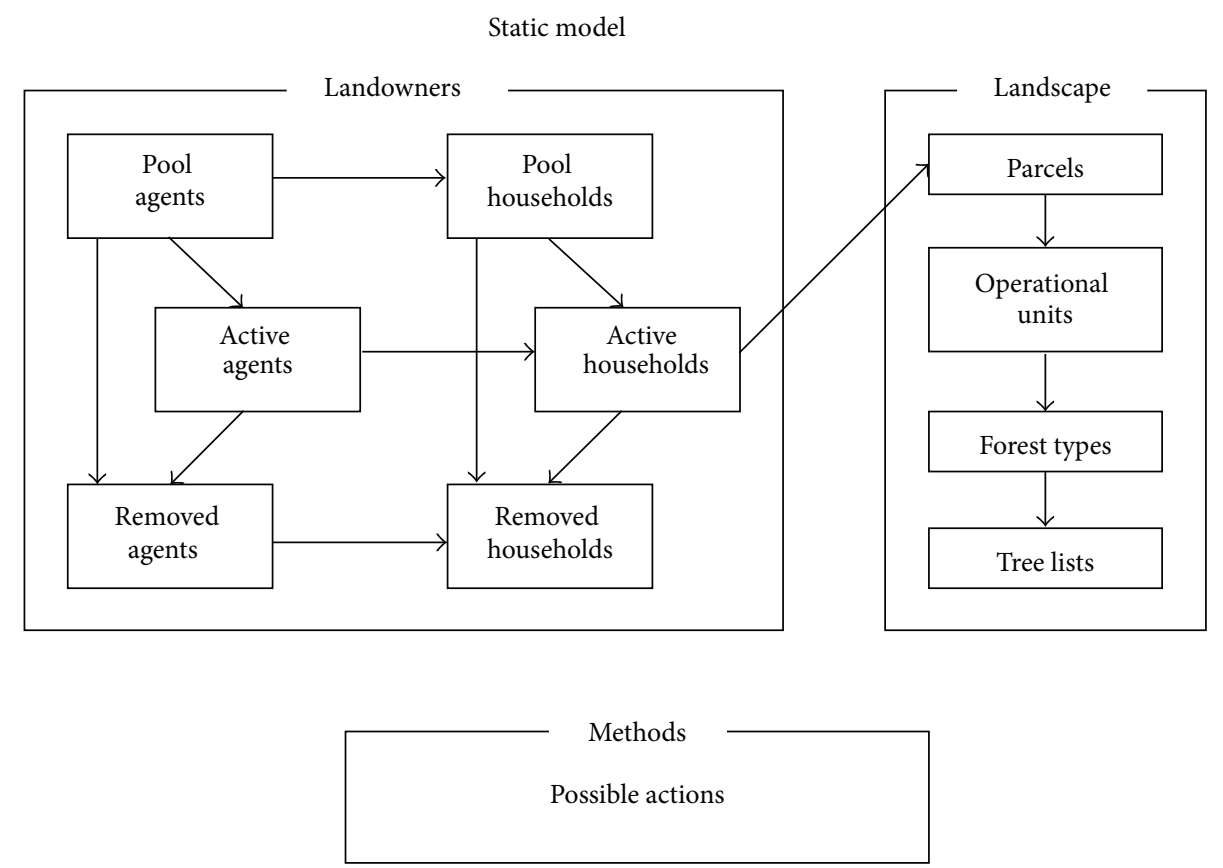

FIgURE 1: The FLAME static constructs. At any given time period, a pool of agents was associated with households. Active agents and households were those that own land. Inactive agents and households were those either deceased or no longer associated with land. The landscape was broken down by multiple units ranging from parcels to tree lists. Methods included actions available to a given agent, such as light or heavy harvesting.

3.3. Process Overview and Scheduling. The static model provided the framework for connecting the landscape and landowners within the model. For the purpose of this model, the landscape was based on GIS data from the rural town of Lincoln, Maine. This town was chosen based on data availability, distribution of small-scale forest parcels, and representativeness of the community to other rural communities in Maine. The model's landscape consisted of all land and water within the model. Landscape was progressively broken down by defined scales ranging from the household level to patches, parcels, and operable units (Figure 1). A parcel was defined as a single unit of land owned by a household as defined by a tax map. In order for a parcel to have harvesting occur, it needed to be at least 4 hectares in size, with a minimum of 4.9 hectares if a residence was present. It was assumed that 0.82 hectares of land would be removed due to the structure of the residence, any associated outbuildings, drives, yard areas, and visual buffers that would likely be placed around such facilities.

Each parcel had a land cover class, such as hardwood, softwood, or mixed-wood, that were associated within given area. This data was derived from the National Land Cover Data. Based on the land cover class, the parcels were populated with stand and tree list data using Forest Inventory and Analysis National Program. The FIA data were selected based on Maine survey plots and generalized to the town of Lincoln, Maine. The parcels were grown over time and harvested by utilizing the USDA Forest Service Forest Vegetation Simulator (FVS).

FLAME assigns the landscape at the parcel level to households. In this case, a household was comprised of
TABLE 1: The FLAME structure data sources by variables.

\begin{tabular}{lcc}
\hline Construct & Variable(s) & Source(s) \\
\hline \multirow{4}{*}{ Landscape } & Parcel boundaries & MEGIS $^{1}$ \\
& Land cover & NLCD $^{2}$ \\
& Stand, tree lists & FIA $^{3}$ \\
& Landscape change & FVS $^{4}$ \\
\hline \multirow{2}{*}{ Landowner } & Demographics & Census $^{5}, \mathrm{PCLOS}^{6}, \mathrm{CDC}^{7}$, \\
& Goals & PCLOS $^{6}, \mathrm{NWOS}^{8}, \mathrm{FGs}^{9}$ \\
& Beliefs & $\mathrm{NWOS}^{8}, \mathrm{FGs}^{9}$ \\
\hline
\end{tabular}

${ }^{1}$ Maine Office of GIS, 2010. ${ }^{2}$ National Land Cover Dataset, 2001. ${ }^{3}$ Forest Inventory and Analysis National Program, 2010. ${ }^{4}$ Forest Vegetation Simulator, United States Department of Agriculture, Forest Service, 2010. ${ }^{5}$ U.S. Census Bureau, 2010. ${ }^{6}$ Penobscot County Landowner Opinion Survey, 2010. ${ }^{7}$ Center for Disease Control, 2010. ${ }^{8}$ National Woodland Owner Survey Table Maker, 2010. ${ }^{9}$ Focus Groups, Small-scale Forest Stakeholder Social Involvement Processes, Gorczyca et al., 2011.

multiple individual agents as landowners. Each agent had their own attributes, such as socioeconomic demographics, goals, and beliefs (Table 1). The socioeconomic attributes of agents were generated based on past survey data, the U.S. Census, or random selections from normal distributions (Table 1).

All modeling components were created using custom Python 2.6.2 code, data storage and relations with Microsoft Access via structured query language (SQL), and landscape change with FVS. One module was created as the model driver, or the main support that ran all intricacies of the 
Dynamic model

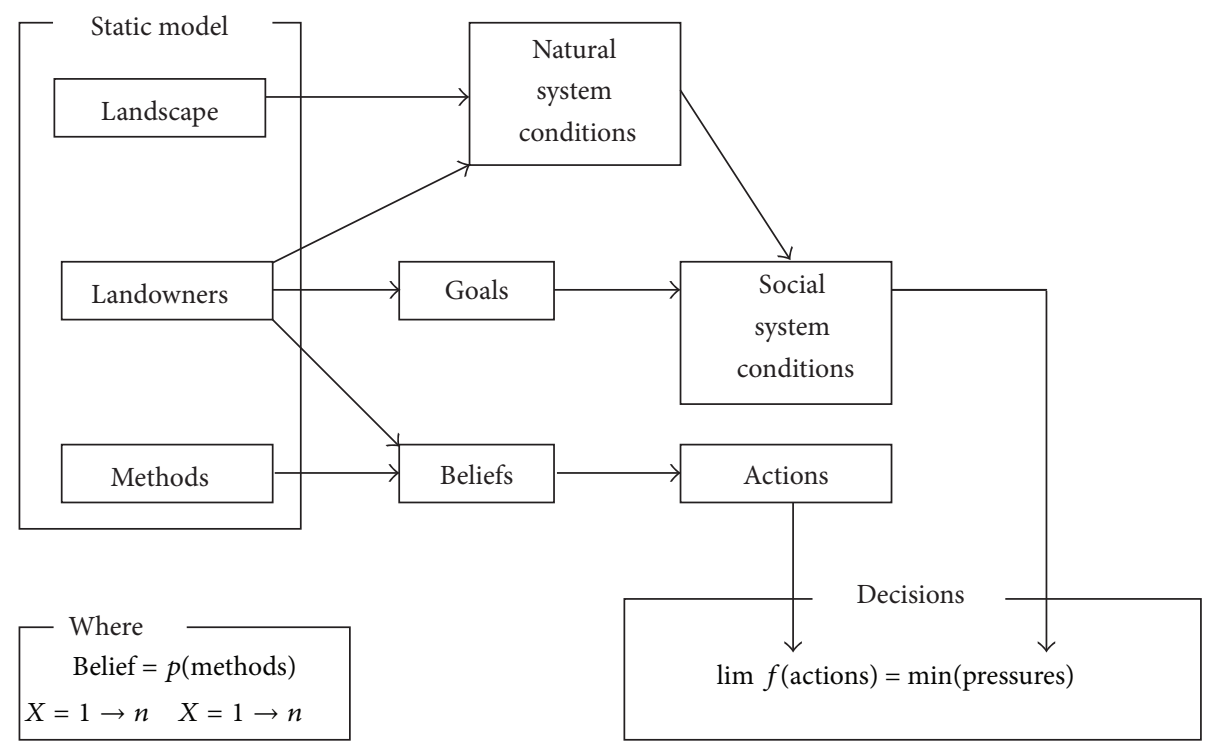

Figure 2: The FLAME dynamic constructs. The static model parameters of landscape, landowner, and methods ultimately drove the dynamic system. Landowners evaluated their natural conditions and priority goal scores as well as communicated. Beliefs interacted with methods (actions available) to represent their perception on how well a given action would meet their goal. The decision engine chose the perceived optimal action that would improve landowners' goal score.

model. The model utilized two databases: an agent database and a support database. As the name implies, the agent database held all information regarding the landowners. Furthermore, as communication, decisions, and actions were performed they were recorded in this database. The support database held all forms of coefficients and other information that were held constant throughout the model simulations. These variables, such as tax rates, were held separately in order to facilitate manipulation of starting variables.

The dynamic model consisted of a series of Python modules that were assembled into a program that progresses the model through time. As the name implies, the dynamic model was where all landowner actions were implemented. These actions then resulted in changes to the agent database each year the model progressed. The key components of the model were landowner beliefs, goals, communication, evaluation, and action decisions (Figure 2). These components are further described as submodels.

For the purpose of understanding small-scale forest timber harvesting behavior, we implemented a one-year time step in the model. This time step advanced not only the agents but also the parcel's trees that were grown and/or cut. The model was simulated on a 25 -year cycle to allow biophysical advancement (trees growth) and social advancement (death, changes in goals, changes in perceptions, etc.) within the model. The scheduling of the processes began with an evaluation of the static model for each agent. Agents would then evaluate their conditions, communicate with other agents, and make decisions. Once agents had decided on actions, the model continued to execute actions and advance the agent population and environment (Figure 3). Each of these steps repeated for each yearly cycle.

\subsection{Design Concepts}

3.4.1. Initialization. At the beginning of the simulation, the baseline database was called upon. This information contained all parameters associated with the landscape, as a compilation of parcels and landowners.

3.4.2. Input. The landscape contained approximately fortyfive hundred parcels and five thousand agents. By creating five thousand starting agents, it was guaranteed there would be sufficient agents to draw from as heirs when the original agents passed away. The probability of death was determined by using actuarial tables and the agent's age.

A total of eight agent goals were defined within the model. Six of these were initially populated according to responses of the Penobscot County Owner Survey and National Woodland Owner Survey. Each goal was summarized based on combining ratings given to personal reasons for owning a parcel of land (Table 2). Responses were on a five-point scale ranging from 1 (very unimportant) to 5 (very important). The scores were calculated by summing the response values for the applicable questions and then dividing by the maximum possible score $(n * 5)$ where $n$ is the number of relevant questions, to produce a score scaled between 0 and 1 . A mean and standard deviation for each of these scores was calculated and used as the basis to randomly generate the scores in the database (Table 2).

A total of twelve beliefs were defined within the model and consisted of harvesting, using a forester, allowing motorized recreation, allowing non-motorized recreation, buying or selling land, subdividing, placing a conservation easement, establishing a family trust, moving, developing 


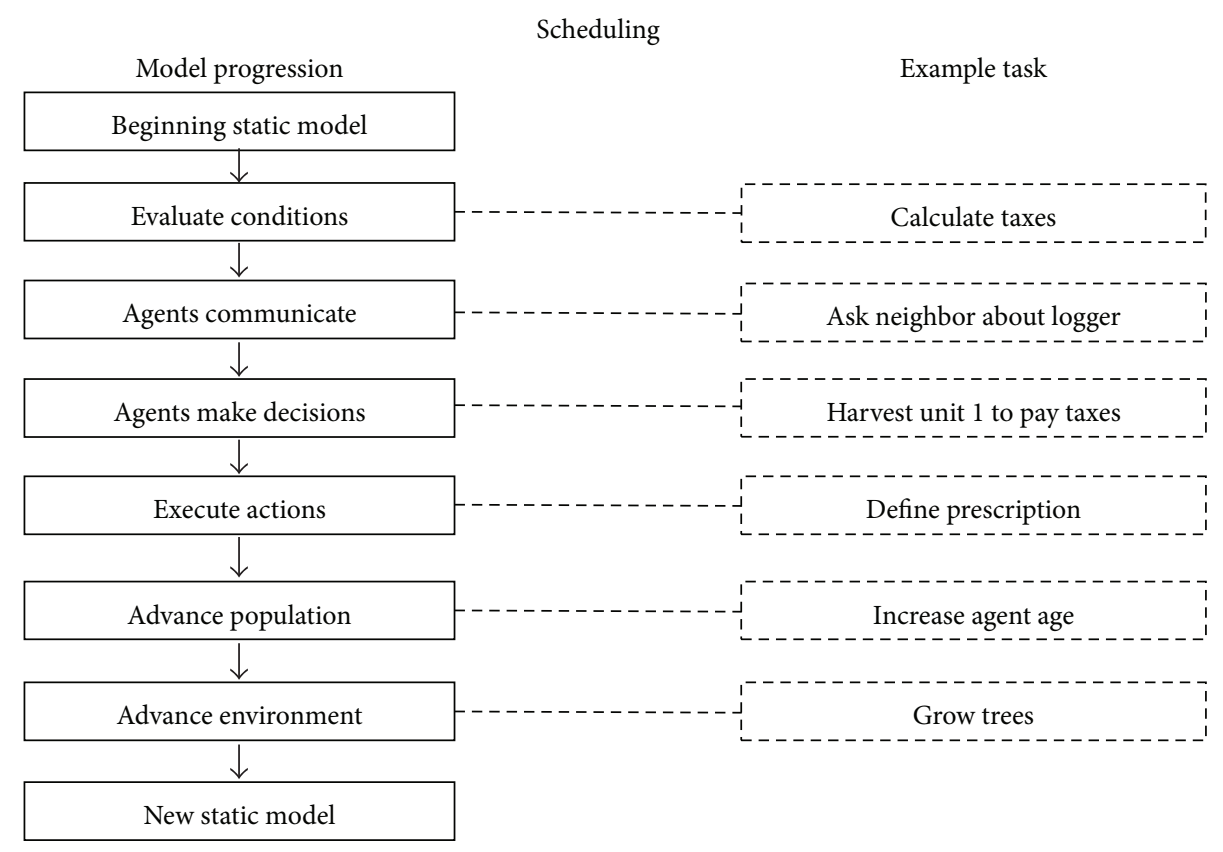

FIGURE 3: The FLAME scheduling process. The model process started with the evaluation of the static model from the previous year. The next steps included communication, decisions, and action execution. The changes in the landscape and landowner parameters were then updated based on action chosen. The end result was a new static model, used to drive the next year.

and building. With the focus on timber, we narrowed the number of beliefs to just those related to harvesting, which corresponded to three possible actions: heavy harvest, light harvest, or no action (Table 3). Each of the beliefs was filled with a decimal value between zero and two using a uniform distribution of the given data range. This range allows positive, negative, and neutral feelings about a belief to be indicated and easily applied through various calculations. A value of one, when multiplied would be neutral, as it would keep everything the same. As the belief value increased from one to two, the multiplication resulted in a stronger positive believe. Conversely, as the belief value decreased from one to zero, the reduced value indicated a negative belief.

3.5. Submodels. The first major component of the model was to evaluate the condition of all of the households. At the start of every cycle, an initial evaluation of the goal equations was conducted. This allowed the model to update the status of all households to reflect any alterations due to actions that occurred in the previous cycle. Eight primary goals were identified to be of potential importance to the agents and their households (Table 2). A separate deterministic function was developed for each goal to determine how well each household was achieving a given goal based on conditions present. The evaluation process compared the actual goals to the desired goal levels. Later, the landowner compared goal discrepancies and made a prioritization or selection of which goal to pursue. After completing the individual score calculations, all goal scores were rescaled in order to allow comparison. While FLAME contained a set of 8 goals, we focus in this manuscript on the fiscal goal score.
After concluding the other goal score evaluations, the model progressed into the financial section of the evaluations. As part of this section, several financial evaluations were conducted before the fiscal score was calculated. The first preparatory calculation was an evaluation of land value. This was necessary because, all land taxes were based on a land valuation. After the land valuation, the next preparatory step in the financial section was to determine any emergency costs. The concept behind emergency costs was to represent stochastic financial burdens, such as a car being wrecked, a medical cost, a house fire, or other events that place a financial burden on the household. The emergency cost was only applied to randomly selected households, until $10 \%$ of agents had some form of emergency cost ranging between $\$ 5,000$ 50,000 . With these two preparatory calculations completed, the model completed the fiscal score calculation for each household. Based on the ratio of debt to wealth, this score represented an indication of the financial wellbeing and fiscal responsibility for each household. The function began by calculating the annual credits for the household, consisting of the total income of the household, the interest from their wealth account, and the previous year's land income. Annual debits included the living costs of adults in the household, home ownership costs, property taxes, income taxes, costs associated with having any children in college, the living costs of any dependent children, emergency costs, and interest accrued on unpaid debts. Annual debits included taxes, the costs associated with having a child in college, the cost of living for number of dependent children, the cost of living for the adults in the household, the costs of a home, emergency costs, income taxes, and interest accrued on unpaid debts. After the calculation of annual credits and debits, the annual 
TABLE 2: The FLAME land ownership goals. A listing of the question responses that were assigned to the initial 6 goals in order to calculate initial goal scores. Intact parcel and home goals were added based on stakeholder recommendations; however, they were populated using similar data distributions as legacy and privacy. The questions were based on the national woodland owner survey regarding reasons for owning land on a 5-point Likert scale: "How important to you are each of the following reasons for owning this parcel?".

\begin{tabular}{ll}
\hline Goals & $\begin{array}{l}\text { Surveyed land ownership reason applied to } \\
\text { goal }\end{array}$ \\
\hline To enjoy the scenery \\
To protect from development \\
To protect wildlife habitat \\
To protect the environment \\
To leave land unmanaged, letting nature \\
take its course
\end{tabular}

net income was calculated by the difference between credit and debit totals. The results for each calculation and final value were stored at the household level for each year in the agent database.

For the purpose of this model, it was assumed an individual landowner was typically unaware of all options available. We proposed that individuals did not have the ability to calculate the impact that every combination of possible decisions would have. Instead, we modeled agent decisions based on a variable named perceptions. Perceptions were the agents' views on how well an action will or will not meet their goal. The perceptions were quantitative estimates of how a given action will alter their wellbeing across the eight goal scores held by each agent. Contained at the household level, these perceptions were created from a summary of the actions and conversations with other agents, where they could be influenced in their perception by the social network within the communication engine.

Perceptions were learned and updated based on the evaluation of recent personal decisions. This was a form of learning, as the agents were taking information from actions they implemented in the past and used this information to
TABLE 3: The enabled beliefs and methods of FLAME. Beliefs were distributed on a scale of $0-2$, from "do not believe in" to "fully believe in" taking a given action. Actions currently enabled in the model range from harvesting and consulting forester to implementing recreation access permission changes. These other beliefs and actions were grouped into a category of no harvest.

\begin{tabular}{lc}
\hline Beliefs & Methods (actions) \\
\hline Harvesting & $\begin{array}{c}\text { Heavy harvest } \\
\text { Light harvest } \\
\text { No harvest }\end{array}$ \\
\hline Using a forester & Consult forester \\
\hline $\begin{array}{l}\text { Allowing motor } \\
\text { recreation }\end{array}$ & Allow motorized recreation \\
\hline $\begin{array}{l}\text { Allowing non-motor } \\
\text { recreation }\end{array}$ & Disallow motorized recreation \\
\hline
\end{tabular}

alter the way in which they would make future decisions. For instance, if no decisions were made in the last yearly cycle there were no changes in perception, if the past action met or exceed the perception, then it created a positive experience, and if the past action did not meet perceptions, or expectations, then it created a negative experience.

After the agents evaluated conditions, calculated goal scores, learned and adjusted perceptions, and communicated; agents then made and implemented decisions. The FLAME incorporated 15 actions available to agents in order to improve goal scores. Of these, we have included only the first 7 actions to simplify harvesting behavior analysis (Table 3 ). The decision engine was a complex string of calculations that chose an action that optimized one or more priority goals as perceived by each household. A household may choose to have more than one action implemented in a year, but the same action type could only be implemented once in a given year. For instance, both a light and a heavy harvest could be implemented in year 1, but each may only happen once during that year. Once the action is implemented, the parcel and household information was updated to reflect all changes. This provided a new static model utilized to drive the next yearly cycle. This process was continued each year until a total of 25 years had been completed.

3.6. Simulation Analysis Methods. The FLAME approach for understanding small-scale forest landowner behavior was used to compare three scenarios of harvesting over a 25 -year period. The first scenario consisted of the baseline parameters with default input values. The second scenario consisted of a socio-economic change within the system, and was implemented at the start of the simulation. To implement this change, we evaluated the land tax structure and estimated a new mill rate assuming the paper mill closed in Lincoln, Maine. This resulted in a new mill rate that was increased by 25 percent in order to retain the same town tax base. Finally, a biophysical change was made within the model to represent a major disturbance event such as a wind or ice storm. To induce this shock, we randomly reduced stands by $0,25,50$, or 75 percent across the entire landscape. This shock 
TABLE 4: Harvesting and goal score ANOVA output. Mean standard deviations (between brackets), sample size and interactions of goal score changes, and hectares harvested by scenario and action. Significant differences in means are bolded.

(a) ANOVA: scenario by action on fiscal goal score

\begin{tabular}{|c|c|c|c|c|c|}
\hline \multirow{2}{*}{ Scenario } & \multirow{2}{*}{ Heavy harvest } & \multirow{2}{*}{ Light harvest } & \multirow{2}{*}{ No harvest } & \multicolumn{2}{|c|}{ Interaction effect } \\
\hline & & & & $F$ & $\rho$ \\
\hline Baseline & $\begin{array}{c}-11623710 \\
(7398318), N=240\end{array}$ & $\begin{array}{c}-6458109 \\
(10513934), N=212\end{array}$ & $\begin{array}{c}-377276 \\
(7904496), N=1229\end{array}$ & 213.08 & 0.000 \\
\hline Socioeconomic & $\begin{array}{c}-12985883 \\
(8321021), N=240\end{array}$ & $\begin{array}{c}-7072157 \\
(13760942), N=211\end{array}$ & $\begin{array}{c}-756019 \\
(8462467), N=1231\end{array}$ & 194.67 & 0.000 \\
\hline Biophysical & $\begin{array}{c}-14304978 \\
(8265848), N=240\end{array}$ & $\begin{array}{c}-10822391 \\
(17840793), N=198\end{array}$ & $\begin{array}{c}-605034 \\
(8013855), N=1236\end{array}$ & 257.83 & 0.000 \\
\hline
\end{tabular}

(b) One-way ANOVA: scenario on total harvested hectares, by heavy, light, and combined

\begin{tabular}{lcccc}
\hline Total hectares & Baseline & Socioeconomic & Biophysical & \multicolumn{2}{c}{ Interaction effect } \\
& & & $F$ & $\rho$ \\
\hline \multirow{2}{*}{ Heavy harvested } & 123.0 & 122.0 & $116.5(52.6)$, & 0.65 \\
& $(73.2)$, & $(75.7)$, & $N=250$ & 0.522 \\
Light harvested & $N=250$ & $N=250$ & 31.3 & 4.98 \\
Combined harvested & $\mathbf{4 1 . 2}$ & 37.0 & $(32.9), N=250$ & 0.007 \\
& $(38.5), N=250$ & $(34.5), N=250$ & $\mathbf{1 4 7 . 8}$ & 3.14 \\
\hline
\end{tabular}

(c) MANOVA: fiscal goal score change by scenario, run, year, action, and interactions $(n=2451)$

\begin{tabular}{lcc}
\hline & & Interaction effect \\
\hline Scenario & $F$ & 0.000 \\
Run & 17.988 & 0.976 \\
Year & 0.297 & 0.000 \\
Action & 23.763 & 0.000 \\
Scenario * action & 875.381 & 0.000 \\
Scenario $*$ year & 7.319 & 0.001 \\
Year $*$ action & 1.795 & 0.000 \\
Run $*$ action & 14.536 & 0.099 \\
\hline
\end{tabular}

(d) MANOVA: total combined hectares harvested by scenario, year, and interactions $(n=336)$

\begin{tabular}{lccc}
\hline & & Interaction effect & $\rho$ \\
\hline Scenario & $F$ & 0.001 \\
Year & 7.179 & 0.000 \\
Scenario $*$ year & 33.415 & 0.001 \\
\hline
\end{tabular}

was implemented prior to the start of the simulation. This was performed manually by subtracting the selected percent reduction $(0,25,50$, or 75 percent $)$ from the total tree count of all stands. Each percentage reduction category was applied to the same amount of stands; a single category was applied to a random 25 percent of the stands within the model. Therefore the disturbance had varied levels of intensity with a total $36.5 \%$ reduction of the tree count. All other variables within each scenario remained unchanged. Each of the 25year scenarios were simulated 10 times, for a total of 30 runs. Harvested hectares and goal score data were extracted and analyzed by year for the three scenarios.

\section{Findings}

4.1. Model Consistency and Validity. After the successful completion of 10 iterative runs per scenario, we first checked for reasonable consistency across runs. This was to ensure that the model was not completely randomized. This analysis included interval plots across runs and checking for significance using statistical tests. We found the plots to have reasonable ranges within the model trend over time, and no statistically significant difference $(\alpha=0.05)$ using ANOVA and MANOVA tests was found among runs in any of the three scenarios (Table 4). This test implies that there is reasonable consistency across iterative runs within each scenario. 


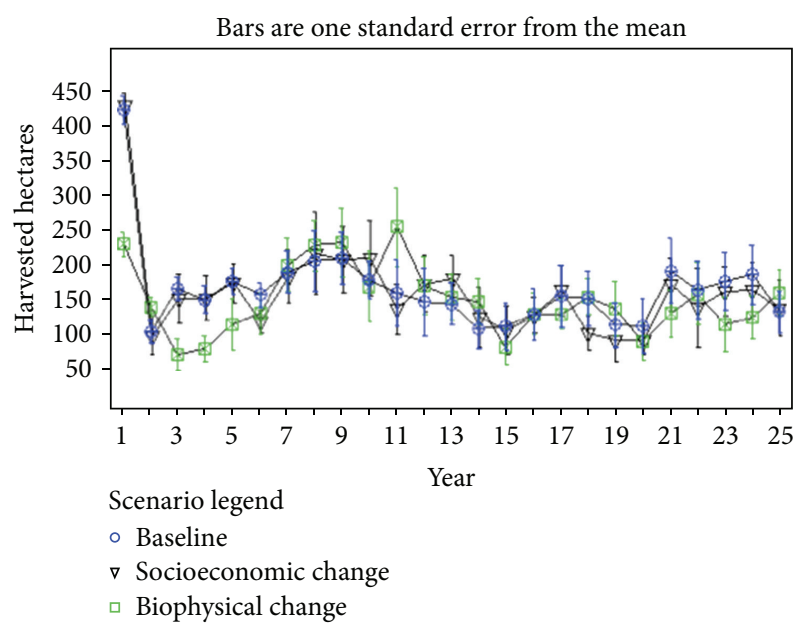

FIGURE 4: Interval plot of total harvested hectares by scenario and year. A 95\% confidence interval plot for the mean of the 10 runs within each scenario of total harvested (combined light and heavy) hectares by year. Here the shapes represent the mean, and the perpendicular lines the interval of one standard error.

After verifying consistency, we evaluated the potential validity of the model by comparing harvesting figures in the baseline scenario with statewide Maine Forest Service data [30]. By summarizing the 1982, 1995, 2003, 2004, and 2006 inventory data for small-scale forest harvests, a total of $1,851,830$ hectares were harvested. This accounted for $20.56 \%$ of small-scale forest land being harvested over a period of 23 years (2004-2006 was averaged to 2005), or $0.82 \%$ of forested hectares harvested per year. In the baseline scenario, a total of 4,105 hectares were harvested from the 13,716 operable hectares of the model. This accounts for $29.93 \%$ of land, with $1.19 \%$ harvested per year. While higher than the statewide figures, we felt comfortable accepting the 1.19\% rate of harvest per year as an indicator of model validity.

4.2. Harvesting Trends by Scenario. To answer the question of whether our socioeconomic and biophysical changes on the system caused significant impact on harvesting behavior, we first analyzed harvested hectares. The total hectares harvested were summarized for all households by year within every run for all three scenarios. Total hectares were assessed by light and heavy harvest treatments, which were also summed to allow further analysis as combined total harvesting. Interval plots of combined harvested, light harvested, and heavy harvested total hectares by scenario and year were produced in MINITAB (Figure 4).

An analysis of variance (ANOVA) was performed on the heavy, light, and combined harvesting data by the three scenarios. Heavy harvesting was not significantly different by scenario $(F=0.65, P=0.522)$. However, light and combined harvesting both had significant differences by scenario, with the baseline being significantly different than the biophysical change scenario. $(F=4.98, P=0.007$ and $F=3.14, P=0.044$; Table 4). We found that for both light and combined harvested hectares, the biophysical change scenario significantly differed from the baseline. Verifying these results, a multiple analysis of variance of scenario, year, and interaction between the two were all found to be significant $(P<0.01)$. This not only suggested variance by scenario, but also through time. Thus there were significant differences between scenario and the year in terms of harvesting. When analyzing the interaction plot by scenario, harvesting patterns of peaks and valleys every few years became apparent (Figure 4). We identified the areas where the biophysical change scenario differed from the other two. Between years 1-5, the biophysical scenario had significantly less total harvested hectares. The biophysical scenario then caught up, and surpassed the other two between years 10-12. After this point, the model began to level out harvesting. These differences can potentially be caused due to the goal score and stand reduction interaction. Furthermore, the rise and falls of the trends across all three scenarios were likely due to the properties of goal score. To better understand this pattern, we also analyzed the fiscal wellness goal.

4.3. Fiscal Goal Score Flux. To answer the question of whether or not landowners were acting to meet their goal, we analyzed the fiscal goal score change by action taken across all three scenarios. In FLAME, goal scores can have very high values when goals are not being met. This means that a large reduction in goal score is an improvement in goal score satisfaction. The change in goal score was calculated by subtracting the second year goal score from the first, the third from the second, and so on. Therefore, large negative goal score change values represent an improved goal score the following year. As before, actions were grouped by heavy harvest, light harvest, and no harvest. Data were again graphed using MINITAB interaction plots to estimate the range of variation of actions within each scenario (Figure 5). Next, several ANOVA tests were utilized to compare scenario fiscal goal scores by actions taken. We found both heavy and light harvest actions to be significantly different than the no harvest action for all three scenarios $(P=0.000)$. This implies that harvesting actions taken improved agent goal scores across all three scenarios. However, no significant difference in fiscal goal score was found between heavy and light harvesting actions $(P>0.05$, Table 4$)$. Since both heavy and light harvesting result in harvest revenue, both improve goal scores. However, the effect of harvest revenue by both heavy and light harvest treatments on the goal score was not statistically different. Furthermore, a multiple analysis of variance was performed to analyze fiscal goal score change by scenario, run, year, action, and interactions between each. All were significant $(P<0.001)$ except for run and run-action interaction $(P=$ $0.099, P=0.967$, Table 4$)$. Having insignificance related to difference among the runs is a sign that the model was reliable within scenario simulations. If there was a significant difference between the runs in the same scenario, we would not be able to compare and contrast scenario trends. The significance across year proves our former statement that there are significant differences in specific years (e.g., years 1-5 for biophysical). 


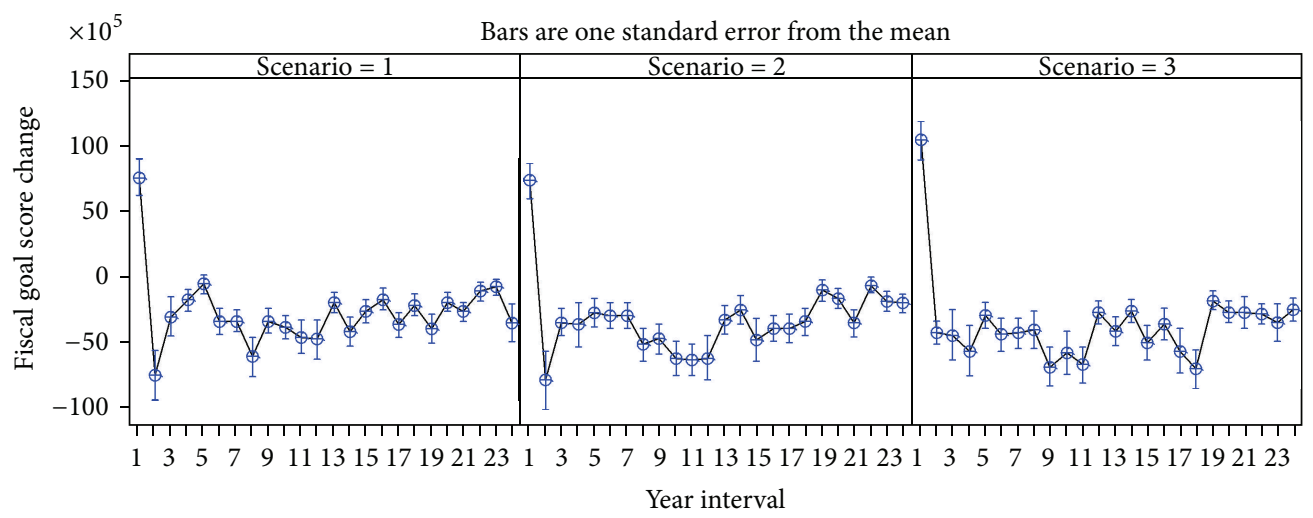

FIgURE 5: Fiscal goal score change interval plots by scenario and year. This graph illustrates the one standard error intervals for the mean of the 10 runs within each of the 3 scenarios: no. 1-baseline, no. 2-socioeconomic, and no. 3-biophysical change. Comparison across year by scenario for goal scores shows the relationship between harvesting actions and their improvement on fiscal goal scores.

\section{Discussion and Conclusions}

As a coupled social-ecological system essential to Maine's economy, small-scale forests proved an intriguing area of study. Their complexities have made predicting behaviors and ecological conditions difficult, especially timber harvesting. New generative social science techniques are especially useful, allowing agent-based modeling to help decipher the interactions between resource conditions and human behavior. This methods section presents FLAME as an ABM-based approach to better understand small-scale forests. Our three system-wide scenarios included a baseline and two shocks to the system: a socioeconomic change of increased taxes and a biophysical change of increased tree mortality. The total harvested hectares by harvest type, model scenario, and yearly time step were analyzed using ANOVA and MANOVA tests. Furthermore, actions and their effect on fiscal goal scores were summarized and also tested using ANOVA and MANOVA. We found a significant difference in harvesting trends by scenario and ultimately found that landowners were taking harvesting actions to meet financial responsibilities.

A significant difference was found in the harvesting trends by scenario. These trends appeared to follow a pattern of peaks and valleys every few years. A few differences should be noted. First, the baseline and socioeconomic scenarios had very high harvesting in the first year. This was likely due to the model "burn in" and adjustment of the input parameters. However, the biophysical scenario does not have as high harvested hectares despite increased tree mortality. It would be reasonable to assume that in a major disturbance event landowners would be salvaging as much as possible. By implementing the shock at the start of the simulation, the standing volume was reduced, thereby leaving less to harvest for the landowners and thus led to lower total harvested hectares. If tree mortality was done in the middle of the simulation, that could allow landowners to recognize the disturbance as an opportunity for salvage harvesting. Instead, it acted as a disturbance similar to that of an ice storm; it kills and damages trees but ultimately does not provide salvage or timber harvesting potential. Despite this, the biophysical scenario quickly reached a larger and longer-lasting peak in harvests than the other two scenarios. Less was available to harvest at the beginning, so landowners with high fiscal need harvested more as time progressed and the stands grew. It is likely that their goal scores reached the point where they had to harvest. This may have implications in our current economy, where tight finances may lead to increased harvesting among small-scale forest landowners in the future. It is possible that many landowners and households have their tipping point where they must obtain cash from their land resources.

The fiscal goal scores corresponded with the trends in harvesting. This contrasts findings by Koontz [10] that economic motivations do not appear to influence harvesting behavior. Increased harvesting corresponded with a significant decrease in the goal score, thus bringing agents closer to meeting their goal. In addition, the effects were significantly different by scenario and action. It makes sense that harvesting (both light and heavy) had an impact on the fiscal goal, since other actions bring less revenue to the agent. Ultimately the analysis of fiscal goal scores allowed the pattern of high and low harvesting trends to emerge. These actions suggest that when landowners face economic hardships, they will consider their land as a financial resource. Erickson et al. [15] found that landowner behavior was mostly unrelated to the timber market prices, and Koontz [10] found no economic motivations for timber harvesting. In contrast, our model was driven by economic motivations and approximated historic harvest levels. This suggested that, perhaps, past research has underestimated the role of economic motivations in predicting small-scale forest harvests. Furthermore, these findings are correlated with the findings of Finley and Kittredge Jr. [18]; landowners who do not have high harvesting values ultimately harvest regardless of their beliefs. Additionally, resource managers must consider the disconnect between landowner intentions and actions when determining the effect of new policy decisions. Although survey responses may give an appearance that landowners do not have harvesting intentions, they ultimately take actions that best suits their financial needs at any given time. 
As with any forecasting model, predicting the future can be very complex to test and verify results. Generally, past data is utilized in order to ascertain the predictive strength of the model. Similarly, we utilized the Maine Forest Service harvest notification data from 1982-2006. The model itself appeared to follow a reasonable amount similarity of harvesting behavior with past trends. However, it is important to note that the harvest notification data was summarized statewide, and not specifically in reference to Lincoln, Maine. Furthermore, harvest notification data has limitations and assumptions, as they are primarily intent to harvest and not a definite date and quantity of harvesting actions. Another limitation of the model is that we have not directly incorporated feedback from landowners themselves. It would be most useful to hold focus groups and conduct surveys of the small-scale forest owners to assess their communication patterns, perceptions, trust, and thoughts about goals and beliefs. This information would provide a means to better understand and represent the baseline parameters within the ABM.

The implication for researchers, forest managers, and policy makers is that $A B M$ is a powerful and useful tool but must be used responsibly. End users must be aware of the assumptions and limitations within the model. ABM should be used to inform their decision-making processes, but not use it as a sole determination. Future studies should continue to enhance ABM analysis techniques in small-scale forests. The ability to model a combination of resource conditions parallel with demographic parameters and generative social science theories creates a new and unprecedented method of understanding small-scale forest behavior. Additional research on baseline parameters, such as landowner communication and perceptions can only improve the results of ABM. In particular, our model included a basic communication engine, with simple social network that allowed agent perceptions to change as a result of "talking" to other agents. Future research should focus on understanding how social networks influence landowner decision-making, and how these influences can be effectively modeled. Advancements to the model could be made by allowing beliefs, goals, and perceptions to be interrelated to each other instead of independent.

Agent-based modeling is still a relatively new field, and this was one of the first attempts to use this technique with small-scale forest landowner timber harvesting behavior. As a result, valuable lessons were learned during our experience of creating and working with FLAME. First, if allowed, agent-based modeling can easily become computationally complex and almost burdensome research technique. FLAME incorporated approximately 11,000 lines of Python code, with Microsoft Access interactions through structured query language (SQL) and the Forest Vegetation Simulator. Second, documentation is especially important for agentbased models (note: the code and full documentation are available from the lead author upon request). Without adequate documentation it is possible to lose track of the exact calculations and model intricacies that create and modify the landowner, landscape, and dynamic operations. Third, while Python is freely available as an open-source coding language, it is relatively slow in terms of processing speeds. Future ABM endeavors may be greatly improved by collaboration with computer scientists capable of writing programs in a faster programming language such as $\mathrm{C}++$. Other programs and even agent-based modeling packages can be useful, but it is important to consider their processing speed and flexibility in terms of modeling parameters. Fourth, another important consideration of agent-based modeling is the available data resources. While we compiled a vast array of data sources into the parameterization of FLAME, we still identified several small-scale forest data gaps. Furthermore, it is important to consider the measurability of the model output. We were fortunate to have strong stakeholder relations with the Maine Forest Service, and obtained harvest notification data. This data allowed us to compare the harvesting trends in Maine with that of FLAME. While this was one method of validation, a more robust analysis could have brought in the NLCD from additional years, and effectively run a set of different land cover dates in the model. This would allow us to better represent the spatial and biophysical components of the landscape through time. Finally, future research would benefit by continuing to include stakeholders in their modeling process.

This study used agent-based modeling in an effort to improve understanding of small-scale forest timber harvesting behavior. We found there is great potential for the use of ABM in small-scale forest resource management as a coupled social-ecological system. It may be a useful approach to not only study land use change but also the motivations and behavior taken by a given small-scale forest landowner [2628]. These findings exemplify the culmination of studies of land use change, resource management, and adaptive forest management into one complex and dynamic model [2629]. As new models are created, the data and understanding of small-scale forests will continue to improve. Researchers and stakeholders will benefit by considering agent-based modeling as a valuable tool with near endless possibilities to increase understanding of small-scale forests and their owners.

\section{Acknowledgments}

We greatly thank Wil Mercier for his contributions to FLAME. This research was supported by the National Science Foundation award EPS-0904155 and Maine EPSCoR at the University of Maine.

\section{References}

[1] A. Hoole and F. Berkes, "Breaking down fences: recoupling social-ecological systems for biodiversity conservation in Namibia," Geoforum, vol. 41, no. 2, pp. 304-317, 2010.

[2] B. J. Butler, E. C. Leatherberry, C. Best, M. A. Kilgore, R. N. Sampson, and K. Larson, "America's family forest owners," Journal of Forestry, vol. 102, no. 7, pp. 4-14, 2004.

[3] B. J. Butler and Z. Ma, "Family forest owner trends in the Northern United States," Northern Journal of Applied Forestry, vol. 28, no. 1, pp. 13-18, 2011.

[4] NWOS Table Maker Version 1. 01, "USDA Forest Service Forest Inventory and Analysis Program National Woodland Owner Survey," 2010, http://apps.fs.fed.us/fia/nwos/tablemaker.jsp. 
[5] K. Laustsen, Personal Conversation with Jessica Leahy. Maine Forest Service Biometrician and State FIA Program Leader, Augusta, Me, USA, 2009.

[6] S. Jin and S. A. Sader, "Effects of forest ownership and change on forest harvest rates, types and trends in northern Maine," Forest Ecology and Management, vol. 228, no. 1-3, pp. 177-186, 2006.

[7] K. M. Laustsen, D. M. Griffith, and J. R. Steinman, "Fourth annual inventory report on Maine's forests," Maine Forest Service, Augusta, Me, USA, 2003, http://www.state.me.us.prxy4 .ursus.maine.edu/doc/mfs/pubs/pdf/anninv/2002invrpt.pdf.

[8] W. H. McWilliams, B. J. Butler, D. M. Griffith, M. L. Hoppus, A. J. Lister, T. W. Lister et al., “The forests of Maine: 2003," Resource Bulletin NE-164, U.S. Department of Agriculture; Forest Service; Northeastern Research Station, Newtown Square, Pa, USA, 2005.

[9] Maine Forest Service, Timber Supply Outlook for Maine: 19952045, Maine Forest Service; Department of Conservation, Augusta, Me, USA, 1998.

[10] T. M. Koontz, "Money talks-but to whom? Financial versus nonmonetary motivations in land use decisions," Society and Natural Resources, vol. 14, no. 1, pp. 51-65, 2001.

[11] J. L. Greene and K. A. Blatner, "Identifying woodland owner characteristics associated with timber management," Forest Science, vol. 32, no. 1, pp. 135-146, 1986.

[12] M. S. Jamnick and D. R. Beckett, "A logit analysis of private woodlot owner's harvesting decisions in New Brunswick," Canadian Journal of Forest Research, vol. 18, no. 3, pp. 330-336, 1988.

[13] J. Kuuluvainen, H. Karppinen, and V. Ovaskainen, "Landowner objectives and nonindustrial private timber supply," Forest Science, vol. 42, no. 3, pp. 300-309, 1996.

[14] L. Lönnstedt, "Non-industrial private forest owners decision process: a qualitative study about goals, time perspective, opportunities and alternatives," Scandinavian Journal of Forest Research, vol. 12, no. 3, pp. 302-310, 1997.

[15] D. L. Erickson, R. L. Ryan, and R. De Young, "Woodlots in the rural landscape: landowner motivations and management attitudes in a Michigan (USA) case study," Landscape and Urban Planning, vol. 58, no. 2-4, pp. 101-112, 2002.

[16] M. G. Rickenbach, D. B. Kittredge, D. Dennis, and T. Stevens, "Ecosystem management: capturing the concept for woodland owners," Journal of Forestry, vol. 96, no. 4, pp. 18-24, 1998.

[17] L. Alexander, Nonindustrial private forest landowner relations to wildlife in New England [Ph.D. thesis], Yale University, New Haven, Conn, USA, 1986.

[18] A. O. Finley and D. B. Kittredge Jr., "Thoreau, Muir, and Jane Doe: different types of private forest owners need different kinds of forest management," Northern Journal of Applied Forestry, vol. 23, no. 1, pp. 27-34, 2006.

[19] D. B. Kittredge Jr., A. O. Finley, and D. R. Foster, “Timber harvesting as ongoing disturbance in a landscape of diverse ownership," Forest Ecology and Management, vol. 180, no. 1-3, pp. 425-442, 2003.

[20] F. W. Cubbage and D. H. Newman, "Forest policy reformed: a United States perspective," Forest Policy and Economics, vol. 9, no. 3, pp. 261-273, 2006.

[21] A. Kangas, S. Laukkanen, and J. Kangas, "Social choice theory and its applications in sustainable forest management-a review," Forest Policy and Economics, vol. 9, no. 1, pp. 77-92, 2006.

[22] V. Grimm, U. Berger, F. Bastiansen et al., "A standard protocol for describing individual-based and agent-based models," Ecological Modelling, vol. 198, no. 1-2, pp. 115-126, 2006.
[23] V. Grimm, U. Berger, D. L. DeAngelis, J. G. Polhill, J. Giske, and S. F. Railsback, "The ODD protocol: a review and first update," Ecological Modelling, vol. 221, no. 23, pp. 2760-2768, 2010.

[24] J. M. Epstein, "Agent-based computational models and generative social science," Complexity, vol. 4, pp. 41-60, 1999.

[25] J. Loomis, C. Bond, and D. Harpman, "The potential of agentbased modelling for performing economic analysis of adaptive natural resource management," Journal of Natural Resources Policy Research, vol. 1, no. 1, pp. 35-48, 2009.

[26] C. Pahl-Wostl and M. Hare, "Processes of social learning in integrated resources management," Journal of Community and Applied Social Psychology, vol. 14, no. 3, pp. 193-206, 2004.

[27] J.-C. Castella, T. N. Trung, and S. Boissau, "Participatory simulation of land-use changes in the northern mountains of Vietnam: the combined use of an agent-based model, a roleplaying game, and a geographic information system," Ecology and Society, vol. 10, no. 1, article 27, 2005.

[28] D. Valbuena, P. H. Verburg, A. K. Bregt, and A. Ligtenberg, "An agent-based approach to model land-use change at a regional scale," Landscape Ecology, vol. 25, no. 2, pp. 185-199, 2010.

[29] E. Gebetsroither, A. Kaufmann, U. Gigler, and A. Resetarits, "Agent-based modelling of self-organisation processes to support adaptive forest management," in Agent-Based Computational Modelling, pp. 153-172, Physica, 2006.

[30] K. Laustsen, Unpublished Harvest Notification Data, Maine Forest Service Biometrician and State FIA Program Leader, Augusta, Me, USA, 2011. 

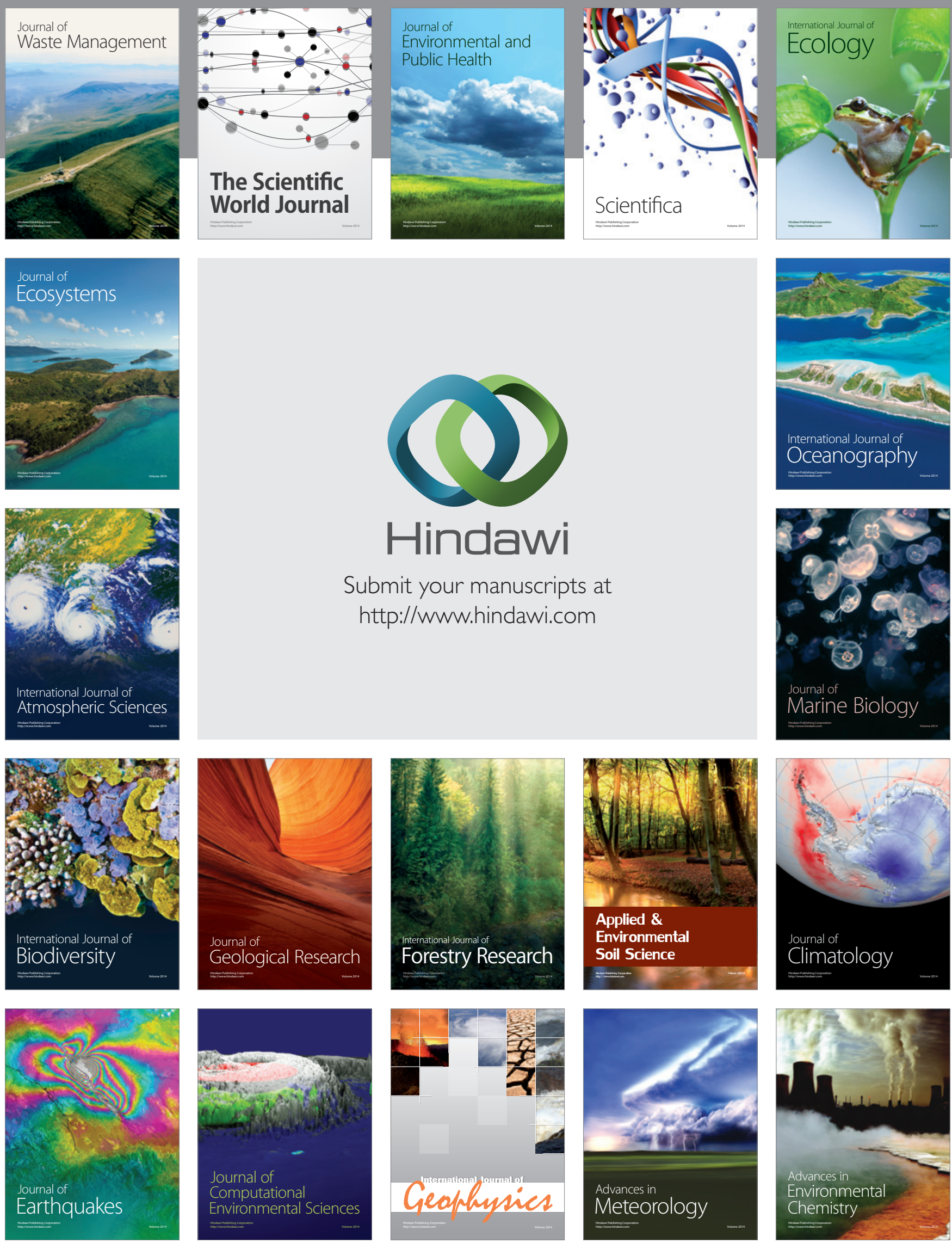